\title{
TESTING FOR DETERMINISTIC AND STOCHASTIC CYCLES IN MACROECONOMIC TIME SERIES
}

\author{
Guglielmo Maria Caporale \\ Brunel University, London
}

\author{
Luis A. Gil-Alana \\ University of Navarra
}

June 2005

\begin{abstract}
In this paper we use a statistical procedure which is appropriate to test for deterministic and stochastic (stationary and nonstationary) cycles in macroeconomic time series. These tests have standard null and local limit distributions and are easy to apply to raw time series. Monte Carlo evidence shows that they perform relatively well in the case of functional misspecification in the cyclical structure of the series. As an example, we use this approach to test for the presence of cycles in US real GDP.
\end{abstract}

Keywords: Deterministic Cycles, Stochastic Cycles, Long Memory

JEL Classification: $\quad$ C22

Corresponding author: Professor Guglielmo Maria Caporale, Brunel Business School, Brunel University, Uxbridge, Middlesex UB8 3PH, UK. Tel.: +44 (0)1895 266713. Fax: +44 (0)1895 269770. Email: Guglielmo-Maria.Caporale@brunel.ac.uk 


\section{Introduction}

It is a well-known stylised fact that many macroeconomic time series can be specified in terms of a trend, and seasonal and cyclical components. However, while the first two of these components have been widely examined in the empirical literature, little attention has been paid to the cyclical structure of the series. In this paper, we focus on the latter, and use an appropriate version of a testing procedure suggested by Robinson (1994) which enables us to test for cyclical structures of any type in a unified framework. These tests have several distinguishing features compared to other procedures. In particular, they have standard null and local limit distributions, implying that it is not necessary to calculate finite sample critical values based on Monte Carlo simulations. In addition, their limiting distribution is the same regardless of the deterministic components used in the regression model, and therefore they are suitable to test for both deterministic and stochastic (stationary or nonstationary) cycles.

The structure of the paper is as follows: Section 2 outlines alternative approaches to modelling cycles in raw time series, and describes the version of the tests of Robinson (1994) used in the present study; Section 3 reports several Monte Carlo experiments aimed at assessing the performance of these tests under misspecification in the functional form of the cycles; Section 4 presents an empirical application to US real GDP, while Section 5 concludes.

\section{Testing for cycles with the tests of Robinson (1994)}

Modelling cycles in macroeconomic time series is still rather controversial. Deterministic cycles based on trigonometric functions of time have been proposed for many years. They are based on models of the form:

$$
y_{t}=\beta_{0} \cos \lambda t+\beta_{1} \sin \lambda t+u_{t}, \quad t=1,2, \ldots
$$


where $\beta_{0}$ and $\beta_{1}$ are fixed parameters, $\lambda$ takes a particular value between 0 and $\pi$, and $u_{t}$ is an $\mathrm{I}(0)$ process, defined for the purpose of the present paper as a covariance stationary process with a spectral density function that is positive and finite at any frequency.

Stochastic stationary cycles were proposed, amongst others, by Harvey (1985). They are based on autoregressive (AR) processes of the form:

$$
y_{t}=\phi_{1} y_{t-1}+\phi_{2} y_{t-2}+u_{t}, \quad t=1,2, \ldots,
$$

with the roots of the AR polynomial lying outside the unit circle. However, in the last few years, it has been claimed that, similarly to the trend and to the seasonal components, cycles may change or evolve over time, and nonstationary stochastic cycles (or unit root cycles) have also been proposed. Thus, for example, Ahtola and Tiao (1987) developed cyclical unit root tests based on the AR(2) model (2), which, under the null hypothesis

$$
\mathrm{H}_{0}:\left|\phi_{1}\right|<2 \text { and } \phi_{2}=-1 \text {, }
$$

becomes the cyclical unit root model specified below. More recently, Gray et al. $(1989,1994)$ extended the cyclical unit root model to the fractional case and considered processes of the form:

$$
\left(1-2 \mu L+L^{2}\right)^{d} x_{t}=u_{t}, \quad t=1,2, \ldots,
$$

where the unit root model corresponds to the case of $d=1$. They showed that the polynomial in (4) can be expressed in terms of the Gegenbauer polynomial such that for all $d \neq 0$

$$
\left(1-2 \mu L+L^{2}\right)^{-d}=\sum_{j=0}^{[T / 2]} C_{j, d}(\mu) L^{j},
$$

where

$$
C_{j, d}(\mu)=\sum_{k=0}^{\infty} \frac{(-1)^{k}(d)_{j-k}(2 \mu)^{j-2 k}}{k !(j-2 k) !} ; \quad(d)_{j}=\frac{\Gamma(d+j)}{\Gamma(d)}
$$

where $\Gamma(\mathrm{x})$ stands for the Gamma function, and a truncation will be required below (5) to make (4) operational. Alternatively, we can use the recursive formula: 


$$
\begin{gathered}
C_{0, d}(\mu)=1, \\
C_{1, d}(\mu)=2 \mu d, \\
C_{j, d}(\mu)=2 \mu\left(\frac{d-1}{j}+1\right) C_{j-1, d}(\mu)-\left(2 \frac{d-1}{j}+1\right) C_{j-2, d}(\mu), \quad j=2,3, \ldots .
\end{gathered}
$$

(see, for instance, Magnus et al., 1966, Rainville, 1960, etc. for further details on Gegenbauer polynomials). Using (5), the process in (4) becomes

$$
x_{t}=\sum_{j=0}^{t-1} C_{j, d}(\mu) u_{t-j}, \quad t=1,2, \ldots
$$

and, when $d=1$, we have

$$
x_{t}=2 \mu x_{t-1}-x_{t-2}+u_{t}, \quad t=1,2, \ldots
$$

which is a cyclical I(1) process with the periodicity determined by $\mu{ }^{1}$ Nesting the unit root cyclical model (7) within the fractional structure (4) has some advantages from a statistical viewpoint. Note that testing (3) in (2) produces a radically different behaviour in the limit distribution. Specifically, if $\phi_{1}$ and $\phi_{2}$ in (2) are such that the roots are within the unit circle, the process is stationary, and the limit distribution is, under appropriate transformations, standard normal; if $\phi_{1}$ and $\phi_{2}$ are given by (3), the process contains unit roots and the limit distribution is non-standard; finally, for the remaining values of $\phi_{1}$ and $\phi_{2}$ the limit distribution is explosive. On the other hand, testing the null of $d=1$ in (4) does not produce such an abrupt change in the limit behaviour, and the boundary line between stationarity and nonstationarity now corresponds to $\mathrm{d}=0.5$ (if $|\mu|<1$ ).

Robinson (1994) developed a general testing procedure which enables one to test all the above specifications for the cyclical structure in a unified framework. He considers the regression model

\footnotetext{
1 Unit root cycles have been examined by Ahtola and Tiao (1987), Chan and Wei (1988), Gregory (1999a, b), and, more recently, by Gil-Alana (2001) and Bierens (2001).
} 


$$
y_{t}=\beta^{\prime} z_{t}+x_{t}, \quad t=1,2, \ldots
$$

where $y_{t}$ is the time series we observe; $\beta$ is a $(k x 1)$ vector of unknown parameters; $z_{t}$ is a $(k x 1)$ vector of exogenous regressors that may include, for example, those in (1); and the regression errors $\mathrm{x}_{\mathrm{t}}$ are of the form given in (4). Thus, we can consider the model

$$
\begin{array}{rr}
y_{t}=\beta_{0} \cos \lambda_{r} t+\beta_{1} \sin \lambda_{r} t+x_{t}, & t=1,2, \ldots \\
\left(1-2 \cos \lambda_{r} L+L^{2}\right)^{d} x_{t}=u_{t}, & t=1,2, \ldots,
\end{array}
$$

where $\lambda_{r}=2 \pi r / T$ and $r=T / j$, $j$ indicating the number of time periods per cycle. Robinson (1994) proposes a Lagrange multiplier (LM) test of the null hypothesis:

$$
\mathrm{H}_{0}: \mathrm{d}=\mathrm{d}_{0}
$$

in (9) and (10) for any real value $d_{0}$. Specifically, the test statistic is given by:

$$
\hat{r}=\frac{T^{1 / 2}}{\hat{\sigma}^{2}} \hat{A}^{-1 / 2} \hat{a}
$$

where $\mathrm{T}$ is the sample size and

$$
\begin{gathered}
\hat{a}=\frac{-2 \pi}{T} \sum_{j}^{*} \psi\left(\lambda_{j}\right) g\left(w_{j} ; \hat{\tau}\right)^{-1} I_{\hat{u}}\left(w_{j}\right) ; \quad \psi\left(w_{j}\right)=\log \left|2\left(\cos w_{j}-\cos \lambda_{r}\right)\right| \\
\hat{A}=\frac{2}{T}\left(\sum_{j}^{*} \psi\left(w_{j}\right) \psi\left(w_{j}\right)^{\prime}-\sum_{j}^{*} \psi\left(w_{j}\right) \hat{\varepsilon}\left(w_{j}\right)^{\prime} x\left(\sum_{j}^{*} \hat{\varepsilon}\left(w_{j}\right) \hat{\varepsilon}\left(w_{j}\right)^{\prime}\right)^{-1} x \sum_{j}^{*} \hat{\varepsilon}\left(w_{j}\right) \psi\left(w_{j}\right)^{\prime}\right) ; \\
\hat{\varepsilon}\left(w_{j}\right)=\frac{\partial}{\partial \tau} \log g\left(w_{j} ; \hat{\tau}\right) ; \quad \hat{\sigma}^{2}=\frac{2 \pi}{T} \sum_{j}^{*} g\left(w_{j} ; \hat{\tau}\right)^{-1} I_{\hat{u}}\left(w_{j}\right) ; \quad w_{j}=\frac{2 \pi j}{T} .
\end{gathered}
$$

$\mathrm{g}\left(\mathrm{w}_{\mathrm{j}} ; \tau\right)$ is the function appearing in the spectral density of $\mathrm{u}_{\mathrm{t}}: \mathrm{f}\left(\mathrm{w}_{\mathrm{j}} ; \tau\right)=\left(\sigma^{2} / 2 \pi\right) \mathrm{g}\left(\mathrm{w}_{\mathrm{j}} ; \tau\right)$, evaluated at $\hat{\tau}=\arg \min \sigma^{2}(\tau)$. Thus, for example, if $\mathrm{u}_{\mathrm{t}}$ is a white noise process, $\mathrm{g} \equiv 1$, whilst if $\mathrm{u}_{\mathrm{t}}$ is an AR process of the form: $\phi(\mathrm{L}) \mathrm{u}_{\mathrm{t}}=\varepsilon_{\mathrm{t}}$, then $\mathrm{g}=\left|\phi\left(\mathrm{e}^{\mathrm{i} \lambda}\right)\right|^{-2}$, with $\sigma^{2}=\mathrm{V}\left(\varepsilon_{\mathrm{t}}\right)$, so that the AR coefficients are a function of $\tau$. Finally, $\mathrm{I}_{\hat{\mathrm{u}}}\left(\mathrm{w}_{\mathrm{j}}\right)$ is the periodogram of $\hat{\mathrm{u}}_{\mathrm{t}}$ defined as:

$$
I_{\hat{u}}\left(\lambda_{j}\right)=\frac{1}{2 \pi T}\left|\sum_{j=1}^{T} \hat{u}_{t} e^{i \lambda_{j} t}\right|^{2} ;
$$




$$
\hat{u}_{t}=\rho(L) y_{t}-\hat{\beta}^{\prime} w_{t} ; \quad w_{t}=\rho(L) z_{t} ; \quad \hat{\beta}=\left(\sum_{t=1}^{T} w_{t} w_{t}{ }^{\prime}\right)^{-1} \sum_{t=1}^{T} w_{t} \rho(L) y_{t},
$$

$\rho(L)=\left(1-2 \cos \lambda_{r} L+L^{2}\right)^{d_{0}}$, and the summations on * in the above expressions are over $\mathrm{w}$ $\in M$, where $M=\left\{w:-\pi<w<\pi, \lambda \notin\left(\rho_{l}-w_{1}, \rho_{l}+w_{1}\right), l=1,2, \ldots, s\right\}$, such that $\rho_{l}, l=1,2, \ldots$, $\mathrm{s}<\infty$ are the distinct poles of $\psi(\mathrm{w})$ on $(-\pi, \pi]$.

Based on $\mathrm{H}_{\mathrm{o}}$ (11), Robinson (1994) established that, under very general conditions,

$$
\hat{r} \rightarrow N(0,1) \quad \text { as } T \rightarrow \infty
$$

and the same limit distribution holds whether or not deterministic regressors are included in (8). Furthermore, he shows that the above test is efficient in the Pitman sense, i.e. that against local alternatives of the form: $\mathrm{H}_{\mathrm{a}}: \mathrm{d}=\mathrm{d}_{\mathrm{o}}+\delta \mathrm{T}^{-1 / 2}$, for $\delta \neq 0$, the limit distribution is normal, with variance 1 and mean that cannot (when $\mathrm{u}_{\mathrm{t}}$ is Gaussian) be exceeded in absolute value by that of any rival regular statistic. Consequently, we are in a classical large sample testing situation for the reasons outlined by Robinson (1994). A one-sided test of $H_{0}$ (11) against the alternative:

$$
\mathrm{H}_{\mathrm{a}}: \mathrm{d}>\mathrm{d}_{\mathrm{o}}
$$

will be given by the rule:

$$
\text { "Reject } \mathrm{H}_{0}(11) \text { if } \hat{r}>\mathrm{z}_{\alpha} \text { ", }
$$

where the probability that a standard normal variate exceeds $z_{\alpha}$ is $\alpha$. Conversely, a test of (11) against the alternative:

$$
\mathrm{H}_{\mathrm{a}}: \mathrm{d}<\mathrm{d}_{\mathrm{o}}
$$

will be given by the rule:

$$
\text { “Reject } H_{o}(11) \text { if } \hat{r}<-z_{\alpha} \text { ". }
$$

Using the set-up described by (9) - (11), we can test for different forms of cyclical structure. For example, if we test $H_{0}(11)$ with $d_{0}=0$ and white noise $u_{t}$, the null model becomes the deterministic structure described in (1); testing the same null hypothesis with 
$\operatorname{AR}(2) u_{t}$, we have a test for stochastic stationary cycles of the form given by (2). Further, testing $\mathrm{H}_{\mathrm{o}}$ with $\mathrm{d}_{0}=1$ amounts to a test for unit root cycles, regardless of whether or not deterministic structures and/or autocorrelated disturbances are included.

\section{Some Monte Carlo evidence}

In this section we examine the finite-sample behaviour of the above version of the tests of Robinson (1994) by means of Monte Carlo simulations. In particular, we investigate their size and power properties in the context of deterministic and stochastic (stationary and nonstationary) cycles. In all cases, we generate Gaussian series using the routines GASDEV and RAN3 of Press, Flannery, Teukolsky and Vetterling (1986), with 10,000 replications. The sample sizes are $\mathrm{T}=60,120,240$ and 360 observations, and the nominal size is $5 \%$.

First, we assume that the cyclical structure of the series is purely deterministic and consider a process of the form:

$$
y_{t}=\cos \lambda_{r} t+\sin \lambda_{r} t+\varepsilon_{t}, \quad t=1,2, \ldots
$$

with $r=T / 6$. We choose this value in view of the fact that cycles in economics seem to occur approximately every six years, and consider alternatives of the form (9) and (10), with $d_{0}=0$, (0.25), 2, and white noise and weakly autocorrelated disturbances.

\section{(Insert Table 1 about here)}

The values reported in Table 1 are the rejection probabilities of the one-sided statistic given by $\hat{r}$ in (12). Hence, the values corresponding to $d_{o}=1$ and white noise $u_{t}$ indicate the size of the tests. One can see that there is a bias in the size in favour of alternatives of the form $\mathrm{H}_{0}: \mathrm{d}<0$, though there is a considerable improvement as the number of observations increases. Specifically, if $T=60$, the sizes are $12.7 \%$ (against $d<0$ ) and $0.9 \%(d>0)$, whilst they improve to 10.3 and $1.9 \%$ respectively when $\mathrm{T}=120$, and to $8.3 \%$ and $3.2 \%$ with $\mathrm{T}=240$. Finally, if $\mathrm{T}=360$, these values become $6.7 \%$ and $4.5 \%$. When the $\mathrm{I}(0)$ disturbances are misspecified, there is a higher distortion in the sizes, although again there is an improvement as 
$\mathrm{T}$ increases. Also, when testing for a unit root (i.e., $\mathrm{d}=1$ ) in this context of deterministic cycles, the rejection probabilities are very high, being equal to 1 in practically all cases if $\mathrm{T}>$ 60.

Next, we assume that the cyclical structure is stochastic, and model the true process in terms of a stationary AR(2) of the form

$$
y_{t}=0.55 y_{t-1}-0.84 y_{t-2}+u_{t}, \quad t=1,2, \ldots .
$$

We choose this parameterisation in order to obtain a cyclical structure with cycles occurring approximately every six periods. Note that the spectral density function of a process like (2) is given by:

$$
\frac{\sigma^{2}}{2 \pi} \frac{1}{\left|1-\phi_{1} e^{i w}-\phi_{2} e^{2 i w}\right|^{2}}=\frac{\sigma^{2}}{2 \pi}\left(\frac{1}{1+\phi_{1}^{2}+\phi_{2}^{2}-2 \phi_{1}\left(1-\phi_{2}\right) \cos w-2 \phi_{2} \cos 2 w}\right)
$$

and setting this expression equal to 0 yields:

$$
w^{*}=\cos ^{-1}\left(\frac{-\phi_{1}\left(1-\phi_{2}\right)}{4 \phi_{2}}\right)
$$

implying that $\mathrm{j}=2 \pi / \mathrm{w}^{*}$. Then, substituting $\phi_{1}$ and $\phi_{2}$ in (18) with, for example, 0.55 and -0.84 , leads to $\mathrm{j} \approx 6$.

\section{(Insert Table 2 about here)}

Table 2 reports the results of the same experiment as in Table 1, but assuming that the true process is generated by (17), while the alternatives are of the form given by (10). It can be seen that, similarly to Table 1 , there is a size distortion with a bias in favour of alternatives with $\mathrm{d}<0$. This distortion is higher than in the previous case of white noise $\mathrm{u}_{\mathrm{t}}$, though again it decreases as $\mathrm{T}$ increases. When the $\mathrm{I}(0)$ disturbances are misspecified, the bias is in the opposite direction, with values close to 0 against $\mathrm{H}_{\mathrm{a}}$ : $\mathrm{d}<0$, and practically equal to 1 if the alternatives are of the form: $d>0$. Moving on to the power of the tests with $d_{0}=1$, the rejection probabilities are close to 1 if $\mathrm{u}_{\mathrm{t}}$ is correctly assumed to be $\mathrm{AR}(2)$, whilst they are relatively low with misspecified disturbances. To sum up, Table 2 seems to suggest that the correct 
specification of the underlying $\mathrm{I}(0)$ autocorrelated disturbances is crucial in the context of stationary stochastic cyclical structures.

\section{(Insert Table 3 about here)}

Finally, we assume that the true data generating process contains cyclical unit roots of the form given by (7) with $\mu=\cos \mathrm{w}_{\mathrm{T} / 6}$ and white noise $\mathrm{u}_{\mathrm{t}}$, and again perform the test in (10) with $d=0$ and 1 . When $d=0$, the rejection probabilities are practically 1 if $u_{t}$ is white noise or $\operatorname{AR}(1)$, and slightly lower if the disturbances are $\operatorname{AR}(2)$. As for the size (i.e., $d=1$ ), once more we observe a bias in favour of alternatives with $d<1$, though, similarly to the previous cases, there is a substantial improvement as the sample size increases.

Overall, the Monte Carlo evidence indicates that the tests of Robinson (1994) are adequate for testing cyclical structures in raw time series, and that, although there is a size distortion when the sample size is small, this tends to disappear as the number of observations increases.

\section{An empirical application}

The time series analysed in this section is the logarithmic transformation of US real GDP in billion dollars, annually, for the time period 1870 -2000, in 1990 prices. Plots of the original series and its first differences, along with their corresponding correlograms and periodograms, are shown in Figure 1. The original series is rising over time, and its nonstationarity is confirmed by the correlogram (with values decaying very slowly), and the periodogram (with a large peak around the zero frequency). Therefore, we perform several unit root tests at the long run or zero frequency. In particular, we use ADF tests (Dickey and Fuller, 1979), where the null hypothesis is that of a unit root in the process; the KPSS test (Kiatwkoswki et al., 1992), for the null of an $\mathrm{I}(0)$ process against the alternative of a unit root; finally, a suitable version of Robinson's (1994) tests (see, e.g., Gil-Alana and Robinson, 1997). In all cases we found evidence of a unit root, and therefore first differences were taken. Their plot suggests that these 
might be stationary, although a cyclical pattern can also be observed; this is especially clear when looking at the correlogram and the periodogram.

\section{(Insert Figure 1 about here)}

Denoting the differenced series by $\mathrm{y}_{\mathrm{t}}$, we use the specification given by (9) and (10) with a constant, i.e., we consider processes of the form

$$
\begin{gathered}
y_{t}=\alpha+\beta_{1} \cos \lambda_{r} t+\beta_{2} \sin \lambda_{r} t+x_{t}, \quad t=1,2, \ldots \\
\left(1-2 \cos \lambda_{r} L+L^{2}\right)^{d} x_{t}=u_{t}, \quad t=1,2, \ldots
\end{gathered}
$$

testing $H_{o}$ (11) with $d_{o}=0$ (in Table 4), and $d_{o}=1$, (in Table 5), and setting $\lambda_{r}=2 \pi r / T, r=T / j$, and $\mathrm{j}=3$, (1), 9, i.e., allowing cycles with a periodicity oscillating between three and nine years. In both Table 4 and 5, we consider separately the cases of (i) $\alpha=\beta_{1}=\beta_{2}=0$ a priori (i.e., we assume no regressors in the levels regression (19)); (ii) $\alpha$ unknown and $\beta_{1}=\beta_{2}=0$ a priori (i.e.., including an intercept); and (iii) all the coefficients unknown, and present the results based on both white noise and weakly (AR) autocorrelated disturbances.

\section{(Insert Tables 4 and 5 about here)}

Table 4 reports the results based on the null hypothesis of $d=0$. Starting with the case with all the coefficients unknown, it can be noticed that the non-rejection values occur when $\mathrm{j}=$ 5 (in the case of white noise $u_{t}$ ), and when $\mathrm{j}=5$ or 6 with AR disturbances; very similar results are obtained if $\beta_{1}=\beta_{2}=0$ a priori or if all the coefficients are 0 . The results based on the null $\mathrm{d}$ $=1$ (in Table 5) decisively reject the hypothesis of cyclical unit roots for all values of $\mathrm{j}$ and all types of disturbances. In fact, the values in Table 5 also indicate that the tests reject the null in favour of alternatives of the form $\mathrm{d}<1$. Note that the tests are based on the statistic given by $\hat{r}$ in (12), and therefore significant negative values represent evidence of orders of integration smaller than 1.

Going back to the results in Table 4, the similarities between the three cases of no regressors, an intercept, and an intercept and cycles may suggest that these deterministic 
components are not required. Gil-Alana and Robinson (1997) introduced a joint test for simultaneously testing the need of a linear time trend and the order of integration at the zero frequency. Here, we propose a similar test, but, instead of looking at the zero frequency, we focus on the cyclical roots, and, instead of a linear trend, we consider a deterministic cyclical structure. Thus, we carry out a joint test of:

$$
H_{o}: d=d_{o} \quad \text { and } \quad \beta=0 \text {, }
$$

with $\beta=\left(\beta_{1}, \beta_{2}\right)$, against alternatives of form:

$$
H_{o}: d \neq d_{o} \quad \text { or } \quad \beta \neq 0
$$

in (19) and (20). This case is not analysed by Robinson (1994), but the LM test can easily be derived as follows:

$$
\tilde{S}=\hat{r}^{2}+\sum_{t=1}^{T} \tilde{u}_{t} w_{2 t}{ }^{\prime} \times\left\{\sum_{t=1}^{T} w_{2 t} w_{2 t}{ }^{\prime}-\sum_{t=1}^{T} w_{2 t} w_{1 t}\left(\sum_{t=1}^{T} w_{1 t}^{2}\right)^{-1} \sum_{t=1}^{T} w_{1 t} w_{2 t}{ }^{\prime}\right\}^{-1} \times \sum_{t=1}^{T} \tilde{u}_{t} w_{2 t},
$$

with $\mathrm{w}_{\mathrm{t}}=\left(\mathrm{w}_{1 \mathrm{t}}, \mathrm{w}_{2 \mathrm{t}}{ }^{\prime}\right)^{\prime}, \mathrm{w}_{1 \mathrm{t}}=\rho(\mathrm{L}) 1_{\mathrm{t}}$ and $\mathrm{w}_{2 \mathrm{t}} \mathrm{t}^{\prime}=\rho(\mathrm{L})\left(\cos \mathrm{w}_{\mathrm{r}} \mathrm{t}, \sin \mathrm{w}_{\mathrm{r}} \mathrm{t}\right)^{\prime}$, and $\tilde{u}_{t}=\left(1-2 \cos w_{r} L+L^{2}\right)^{d_{o}} y_{t}-\tilde{\alpha} w_{1 t} ; \tilde{\alpha}=\left(\sum_{t=1}^{T} w_{1 t}^{2}\right)^{-1} \sum_{t=1}^{T} w_{1 t}\left(1-2 \cos w_{r} L+L^{2}\right)^{d_{o}} y_{t}$

and $\hat{r}$ calculated as described in Section 2 but using the $\tilde{u}_{t}$ just defined. Then, under $\mathrm{H}_{\mathrm{o}}(21)$, $\tilde{S} \rightarrow{ }_{d} \chi_{3}^{2}$ as $\mathrm{T} \rightarrow \infty$, and (23) is compared to the values of the upper tail of the $\chi_{3}^{2}$ distribution.

\section{(Insert Table 6 about here)}

In Table 6 we present the statistic (23) for the same values of $\mathrm{j}$ and $\mathrm{d}_{\mathrm{o}}$ as before. It can be seen that, similarly to the previous tables, the non-rejection values occur when $\mathrm{j}=5$ and 6 , implying that deterministic cycles may not be important when modelling this series. In view of all this evidence, and also taking into account the insignificance of the estimated coefficients in the models based on an intercept and on AR(1) disturbances, we can conclude that the best model specification for the growth rate series is a stationary AR(2) model of the form given by 
(2), with estimated coefficients equal to 0.278 and -0.072 . According to (18), this implies that the cycles occur approximately every 6.29 periods, which is consistent with the empirical evidence for many other macroeconomic time series.

\section{Conclusions}

In this paper we have presented a version of the tests of Robinson (1994) that is appropriate to test for cyclical components in raw time series. These tests are very general and suitable for both deterministic and stochastic (stationary and nonstationary) cycles without any change in their standard (normal) limit distribution. We report several Monte Carlo experiments showing that their size is slightly biased for small sample sizes, but approximates the nominal one for higher values of $\mathrm{T}$. Also, we show that these tests have power to detect functional misspecification in the cyclical case. Finally, we applied them to the first differences of the log transformation of US real GDP. We find strong evidence against unit root cycles, and deterministic components also seem to be inappropriate. A simple AR(2) process with stationary complex roots appears to be the best specification for describing the cyclical structure of this series.

The present study can be extended in several ways. For instance, finite-sample critical values for the different forms of cyclical structures could be computed, and the case of nonnormal disturbances could also be considered. Further, it might be of interest to obtain point estimates of the fractional differencing parameters for the cyclical components (examples in a semiparametric context are Arteche and Robinson, 2000, and Arteche, 2001). However, this would be much more computationally intensive. Moreover, the emphasis should be put on confidence intervals, rather than point estimates, when preliminary integer differencing appears to be required in order to achieve I(0) stationarity. ${ }^{2}$

\footnotetext{
${ }^{2}$ Note that the approach used in this paper generates simply computed diagnostics for departures from a particular type of cyclical behaviour; specifically, the results presented in this paper should be interpreted as giving support to models assuming that real output is I(1) with the cyclical structure determined by a stationary AR(2) model.
} 


\section{References}

Ahtola, J.. and Tiao, G.C., 1987, Distributions of least squares estimators of autoregressive parameters for a process with complex roots on the unit circle, Journal of Time Series Analysis 8, 1-14.

Arteche, J. and P.M. Robinson, Seasonal and cyclical long memory, in S. Ghosh ed., Asymptotics, Nonparametrics and Time Series, Marcel Dekker Inc., New York, 115-148.

Arteche, J. and P.M. Robinson, 2000, Semiparametric inference in seasonal and cyclical long memory processes, Journal of Time Series Analysis 21, 1-25.

Bierens, H.J., 2001, Complex unit roots and business cycles, Are they real?, Econometric Theory 17, 962-983.

Chan, Y. and C.Z. Wei, 1988, Limiting distributions of least squares estimates of unstable autoregressive process, Annals of Statistics 16, 367-401.

Dickey D.A. and W.A. Fuller, 1979, Distribution of the estimators for autoregressive time series with a unit root, Journal of the American Statistical Association 74, 427-431.

Gil-Alana, L.A., 2000, Mean reversion in the real exchange rates, Economics Letters 69, 285288.

Gil-Alana, L.A. and Robinson, P.M., 1997, Testing of unit roots and other nonstationary hypotheses in macroeconomic time series, Journal of Econometrics 80, 241-268.

Gray, H.L., Yhang, N. and Woodward, W.A., 1989, On generalized fractional processes, Journal of Time Series Analysis 10, 233-257.

Gray, H.L., Yhang, N. and Woodward, W.A., 1994, On generalized fractional processes. A correction, Journal of Time Series Analysis 15, 561-562.

Gregoir, S., 1999a, Multivariate time series with various hidden unit roots. Part I. Integral operator algebra and representation theory, Econometric Theory 15, 435-468.

Gregoir, S., 1999b, Multivariate time series with various hidden unit roots. Part II. Estimation and testing, Econometric Theory 15, 469-518.

Harvey, A., 1985, Trends and cycles in macroeconomic time series, Journal of Business and Economics Statistics 3, 216-227.

Kwiatkowski, D., P.C.B. Phillips, P. Schmidt and Y. Shin, 1992, Testing the null hypothesis of stationarity against the alternative of a unit root, Journal of Econometrics 54, 159-178.

Magnus, W., Oberhettinger, F. and R.P. Soni, 1966, Formulas and theorema for the special functions of mathematical physics, Springer, Berlin.

Press, W.H., Flannery, B.P., Teukolsky, S.A. and Vetterling, W.T., 1986, Numerical recipes: The art of scientific computing, Cambridge University Press, Cambridge. 
Rainville, E.D., 1960, Special functions, MacMillan, New York.

Robinson, P.M., 1994, Efficient tests of nonstationary hypotheses, Journal of the American Statistical Association 89, 1420-1437. 


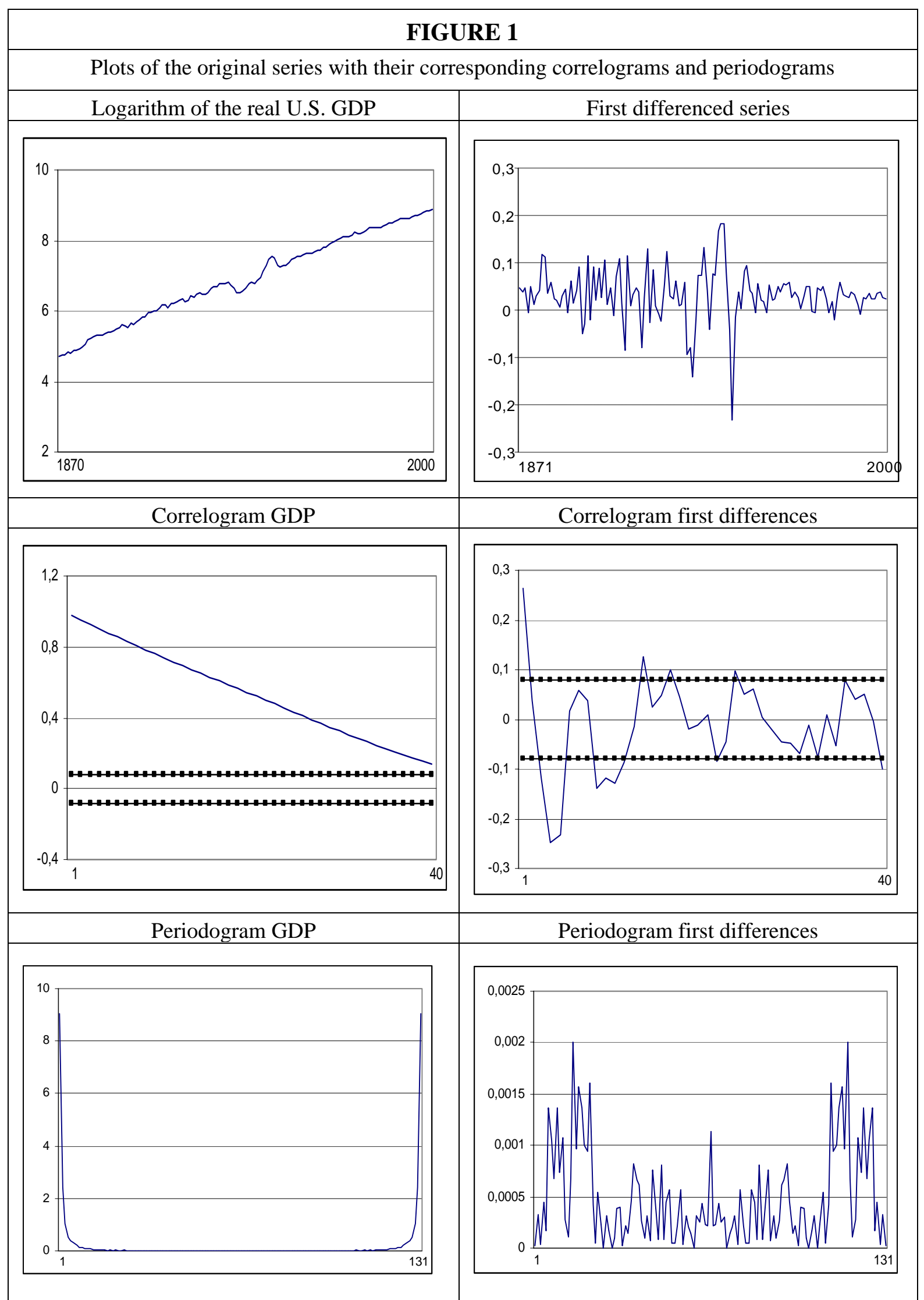

The large sample standard error under the null hypothesis of no autocorrelation is $1 / \sqrt{ } \mathrm{T}$ or roughly 0.08 for the series used in this application. 
TABLE 1

\section{Rejection frequencies of the tests of Robinson (1994) in the context of deterministic cyclical structures}

True model: $y_{t}=\cos \lambda_{T / 6} t+\sin \lambda_{T / 6} t+\varepsilon_{t}$, Alternatives: $\quad y_{t}=\beta_{0} \cos \lambda_{T / 6} t+\beta_{1} \sin \lambda_{T / 6} t+x_{t}$,

$$
\left(1-2 \cos \lambda_{T / 6} L+L^{2}\right)^{d} x_{t}=u_{t} \text {, }
$$

\begin{tabular}{|c|c|c|c|c|}
\hline \multirow{2}{*}{ Sample size } & \multirow{2}{*}{ Disturbances } & \multicolumn{2}{|c|}{$\mathrm{H}_{0}: \mathrm{d}=0$} & $\mathrm{H}_{0}: \mathrm{d}=1$ \\
\cline { 2 - 5 } & & $\mathrm{H}_{\mathrm{o}}: \mathrm{d}<0$ & $\mathrm{H}_{0}: \mathrm{d}>0$ & $\mathrm{H}_{0}: \mathrm{d}<1$ \\
\hline \multirow{2}{*}{$\mathrm{T}=60$} & White noise & $\mathbf{0 . 1 2 7}$ & $\mathbf{0 . 0 0 9}$ & 1.000 \\
\cline { 2 - 5 } & AR(1) & 0.152 & 0.004 & 0.993 \\
\cline { 2 - 5 } & AR(2) & 0.164 & 0.003 & 0.987 \\
\hline \hline \multirow{2}{*}{$\mathrm{T}=120$} & White noise & $\mathbf{0 . 1 0 3}$ & $\mathbf{0 . 0 1 9}$ & 1.000 \\
\cline { 2 - 5 } & AR(1) & 0.136 & 0.016 & 1.000 \\
\cline { 2 - 5 } & AR(2) & 0.149 & 0.012 & 0.999 \\
\hline \hline \multirow{2}{*}{$\mathrm{T}=240$} & White noise & $\mathbf{0 . 0 8 3}$ & $\mathbf{0 . 0 3 2}$ & 1.000 \\
\cline { 2 - 5 } & AR(1) & 0.099 & 0.026 & 1.000 \\
\cline { 2 - 5 } & AR(2) & 0.105 & 0.024 & 1.000 \\
\hline \hline \multirow{2}{*}{$\mathrm{T}=360$} & White noise & $\mathbf{0 . 0 6 7}$ & $\mathbf{0 . 0 4 5}$ & 1.000 \\
\cline { 2 - 5 } & AR(1) & 0.071 & 0.044 & 1.000 \\
\cline { 2 - 5 } & AR(2) & 0.073 & 0.043 & 1.000 \\
\hline \hline
\end{tabular}

In bold: The sizes of the tests. The nominal size is $95 \%$ and 10,000 replications were carried out in each case. 
TABLE 2

Rejection frequencies of the tests of Robinson (1994) in the context of stationary stochastic cyclical structures

True model: $y_{t}=0.55 y_{t-1}-0.84 y_{t-2}+\varepsilon_{t}$,

Alternatives: $\left(1-2 \cos \lambda_{r} L+L^{2}\right)^{d} y_{t}=u_{t}$,

\begin{tabular}{|c|c|c|c|c|}
\hline \multirow{2}{*}{ Sample size } & \multirow{2}{*}{ Disturbances } & \multicolumn{2}{|c|}{$\mathrm{H}_{0}: \mathrm{d}=0$} & $\mathrm{H}_{0}: \mathrm{d}=1$ \\
\cline { 2 - 5 } & & $\mathrm{H}_{0}: \mathrm{d}<0$ & $\mathrm{H}_{0}: \mathrm{d}>0$ & $\mathrm{H}_{0}: \mathrm{d}<1$ \\
\hline \multirow{2}{*}{$\mathrm{T}=60$} & White noise & 0.000 & 0.996 & 0.365 \\
\cline { 2 - 5 } & AR(1) & 0.000 & 0.988 & 0.317 \\
\cline { 2 - 5 } & AR(2) & $\mathbf{0 . 2 6 1}$ & $\mathbf{0 . 0 0 0}$ & 0.895 \\
\hline \hline \multirow{2}{*}{$\mathrm{T}=120$} & White noise & 0.000 & 1.000 & 0.512 \\
\cline { 2 - 5 } & AR(1) & 0.000 & 1.000 & 0.506 \\
\cline { 2 - 5 } & AR(2) & $\mathbf{0 . 1 9 8}$ & $\mathbf{0 . 0 0 4}$ & 0.9976 \\
\hline \hline \multirow{2}{*}{$\mathrm{T}=240$} & White noise & 0.000 & 1.000 & 0.677 \\
\cline { 2 - 5 } & AR(1) & 0.000 & 1.000 & 0.605 \\
\cline { 2 - 5 } & AR(2) & $\mathbf{0 . 0 9 7}$ & $\mathbf{0 . 0 1 5}$ & 1.000 \\
\hline \hline \multirow{2}{*}{$\mathrm{T}=360$} & White noise & 0.000 & 1.000 & 0.775 \\
\cline { 2 - 5 } & AR(1) & 0.000 & $\mathbf{0 . 0 2 2}$ & 0.707 \\
\cline { 2 - 5 } & AR(2) & $\mathbf{0 . 0 8 3}$ & 1.000 & 1.000 \\
\hline \hline
\end{tabular}

In bold: the sizes of the tests. The nominal size is $95 \%$ and 10,000 replications were carried out in each case. 
TABLE 3

Rejection frequencies of the tests of Robinson (1994) in the context of nonstationary stochastic cyclical structures

True model: $y_{t}=2 \cos \lambda_{T / 6} y_{t-1}-y_{t-2}+\varepsilon_{t}$,

\begin{tabular}{|c|c|c|c|c|}
\hline \multicolumn{5}{|c|}{ Alternatives: $\left(1-2 \cos \lambda_{T / 6} L+L^{2}\right)^{d} y_{t}=u_{t}}$, \\
\hline \multirow{2}{*}{ Sample size } & Disturbances & $\mathrm{H}_{0}: \mathrm{d}=0$ & \multicolumn{2}{c|}{$\mathrm{H}_{0}: \mathrm{d}=1$} \\
\cline { 2 - 5 } & & $\mathrm{H}_{0}: \mathrm{d} \neq 0$ & $\mathrm{H}_{0}: \mathrm{d}<1$ & $\mathrm{H}_{0}: \mathrm{d}>1$ \\
\hline \multirow{2}{*}{$\mathrm{T}=60$} & White noise & 0.763 & $\mathbf{0 . 1 3 6}$ & $\mathbf{0 . 0 1 5}$ \\
\cline { 2 - 5 } & AR(1) & 0.768 & 0.141 & 0.002 \\
\cline { 2 - 5 } & AR(2) & 0.711 & 0.468 & 0.004 \\
\hline \hline \multirow{2}{*}{$\mathrm{T}=120$} & White noise & 0.991 & $\mathbf{0 . 1 0 2}$ & $\mathbf{0 . 0 2 2}$ \\
\cline { 2 - 5 } & AR(1) & 0.995 & 0.144 & 0.006 \\
\cline { 2 - 5 } & AR(2) & 0.833 & 0.441 & 0.033 \\
\hline \hline \multirow{2}{*}{$\mathrm{T}=240$} & White noise & 1.000 & $\mathbf{0 . 0 8 0}$ & $\mathbf{0 . 0 3 9}$ \\
\cline { 2 - 5 } & AR(1) & 1.000 & 0.174 & 0.026 \\
\cline { 2 - 5 } & AR(2) & 0.859 & 0.515 & 0.113 \\
\hline \hline \multirow{2}{*}{$\mathrm{T}=360$} & White noise & 1.000 & $\mathbf{0 . 0 6 1}$ & $\mathbf{0 . 0 4 8}$ \\
\cline { 2 - 5 } & AR(1) & 1.000 & 0.173 & 0029 \\
\cline { 2 - 5 } & AR(2) & 0.984 & 0.546 & 0.110 \\
\hline \hline
\end{tabular}

In bold: The sizes of the tests. The nominal size is $95 \%$ and 10,000 replications were carried out in each case. 
TABLE 4

Testing of stationary cycles with the tests of Robinson (1994)

Model: $\quad y_{t}=\alpha+\beta_{0} \cos \lambda_{j} t+\beta_{1} \sin \lambda_{j} t+x_{t}$,

$$
\left(1-2 \cos \lambda_{j} L+L^{2}\right)^{d} x_{t}=u_{t}, \quad \mathrm{H}_{0}: \mathrm{d}=0
$$

\begin{tabular}{|c|c|c|c|c|c|c|c|}
\hline $\mathrm{U}_{\mathbf{t}} / \mathrm{j}$ & 3 & 4 & 5 & 6 & 7 & 8 & 9 \\
\hline White noise & -3.06 & -1.70 & $\mathbf{0 . 1 2}$ & 1.90 & 3.47 & 4.10 & 3.38 \\
\hline $\mathrm{AR}(1)$ & -2.05 & -1.77 & $\mathbf{- 1 . 0 2}$ & $\mathbf{- 0 . 5 2}$ & 2.31 & 3.65 & 3.40 \\
\hline $\mathrm{AR}(2)$ & -3.34 & -1.98 & $\mathbf{- 1 . 6 5}$ & $\mathbf{- 1 . 3 9}$ & 2.04 & 3.11 & 3.54 \\
\hline \hline
\end{tabular}

Imposing $\beta_{0}=\beta_{1}=0$

\begin{tabular}{|c|c|c|c|c|c|c|c|}
\hline $\mathrm{U}_{\mathrm{t}} / \mathrm{j}$ & 3 & 4 & 5 & 6 & 7 & 8 & 9 \\
\hline White noise & -2.92 & $-1.56^{\prime}$ & $\mathbf{0 . 1 2}$ & 2.20 & 3.88 & 4.40 & 3.99 \\
\hline $\mathrm{AR}(1)$ & -2.17 & -1.71 & $\mathbf{- 1 . 0 2}$ & $\mathbf{- 0 . 2 3}$ & 3.08 & 3.65 & 3.11 \\
\hline $\mathrm{AR}(2)$ & -3.45 & -1.68 & $\mathbf{- 1 . 3 9}$ & $\mathbf{- 0 . 7 3}$ & 3.34 & 3.21 & 2.95 \\
\hline \hline
\end{tabular}

Imposing $\alpha=\beta_{0}=\beta_{1}=0$

\begin{tabular}{|c|c|c|c|c|c|c|c|}
\hline $\mathrm{U}_{\mathrm{t}} / \mathrm{j}$ & 3 & 4 & 5 & 6 & 7 & 8 & 9 \\
\hline $\mathrm{AR}(1)$ & -2.99 & -1.71 & $\mathbf{- 1 . 0 3} \mathbf{\prime}^{\prime}$ & $\mathbf{- 0 . 2 3}$ & 2.31 & 3.21 & 3.90 \\
\hline $\mathrm{AR}(2)$ & -3.21 & -1.69 & $\mathbf{- 1 . 4 0}$ & $\mathbf{- 0 . 7 4}$ & 1.98 & 2.34 & 2.98 \\
\hline
\end{tabular}

' and in bold: Non-rejection values of the null hypothesis at the 95\% significance level. 
TABLE 5

Testing of nontationary (integrated) cycles with the tests of Robinson (1994)

Model: $y_{t}=\alpha+\beta_{0} \cos \lambda_{j} t+\beta_{1} \sin \lambda_{j} t+x_{t}$,

$$
\left(1-2 \cos \lambda_{j} L+L^{2}\right)^{d} x_{t}=u_{t}, \quad \mathrm{H}_{0}: \mathrm{d}=1
$$

\begin{tabular}{|c|c|c|c|c|c|c|c|}
\hline $\mathrm{U}_{\mathrm{t}} / \mathrm{j}$ & 3 & 4 & 5 & 6 & 7 & 8 & 9 \\
\hline White noise & -7.87 & -4.13 & -4.67 & -6.64 & -7.90 & -8.58 & -8.96 \\
\hline $\mathrm{AR}(1)$ & -8.54 & -4.32 & -11.98 & -7.08 & -8.08 & -8.34 & -7.09 \\
\hline $\mathrm{AR}(2)$ & -6.59 & -4.40 & -16.44 & -15.06 & -10.04 & -9.07 & -7.85 \\
\hline \hline \multicolumn{7}{|c|}{ Imposing $\beta_{0}=\beta_{1}=0$} \\
\hline $\mathrm{U}_{\mathrm{t}} / \mathrm{j}$ & 3 & 4 & 5 & 6 & 7 & 8 & 9 \\
\hline White noise & -7.89 & -4.16 & -4.69 & -6.66 & -7.91 & -8.59 & -8.96 \\
\hline $\mathrm{AR}(1)$ & -7.99 & -5.26 & -11.13 & -7.13 & -8.00 & -8.34 & -7.11 \\
\hline $\mathrm{AR}(2)$ & -8.04 & -4.39 & -16.54 & -15.67 & -8.91 & -9.08 & -7.88 \\
\hline \hline \multicolumn{7}{|c|}{ Imposing $\alpha=\beta_{0}=\beta_{1}=0$} \\
\hline $\mathrm{U}_{\mathrm{t}} / \mathrm{j}$ & 3 & 4 & 5 & 6 & 7 & 8 & 9 \\
\hline White noise & -7.89 & -4.16 & -4.69 & -6.66 & -7.91 & -8.58 & -8.97 \\
\hline $\mathrm{AR}(1)$ & -8.43 & -5.26 & -11.12 & -7.23 & -7.88 & -8.34 & -7.09 \\
\hline $\mathrm{AR}(2)$ & -8.04 & -4.40 & -16.56 & -15.98 & -8.90 & -9.08 & -7.88 \\
\hline
\end{tabular}


TABLE 6

Joint test of (21) against (22) in model given by (19) and (20) and white noise $u_{t}$

\begin{tabular}{|c|c|c|}
\hline $\mathrm{J}$ & $\mathrm{H}_{0}: \mathrm{d}=0$ and $\beta=0$ & $\mathrm{H}_{0}: \mathrm{d}=1$ and $\beta=0$ \\
\hline 3 & 10.58 & 62.30 \\
\hline 4 & 8.46 & 17.31 \\
\hline 5 & $\mathbf{0 . 0 1} \boldsymbol{2}$ & 44.07 \\
\hline 6 & $\mathbf{4 . 8 6}$ & 62.69 \\
\hline 7 & 15.21 & 73.82 \\
\hline 8 & 19.43 & 80.45 \\
\hline 9 & 15.98 & \\
\hline
\end{tabular}

' and in bold: Non-rejection values for the null hypothesis at 95\% significance level. 